\title{
Calibration of the ATLAS Muon Spectrometer Precision Chambers for Initial LHC Beam Collisions
}

\section{Haijun Yang}

University of Michigan, Ann Arbor

on behalf of the ATLAS Collaboration

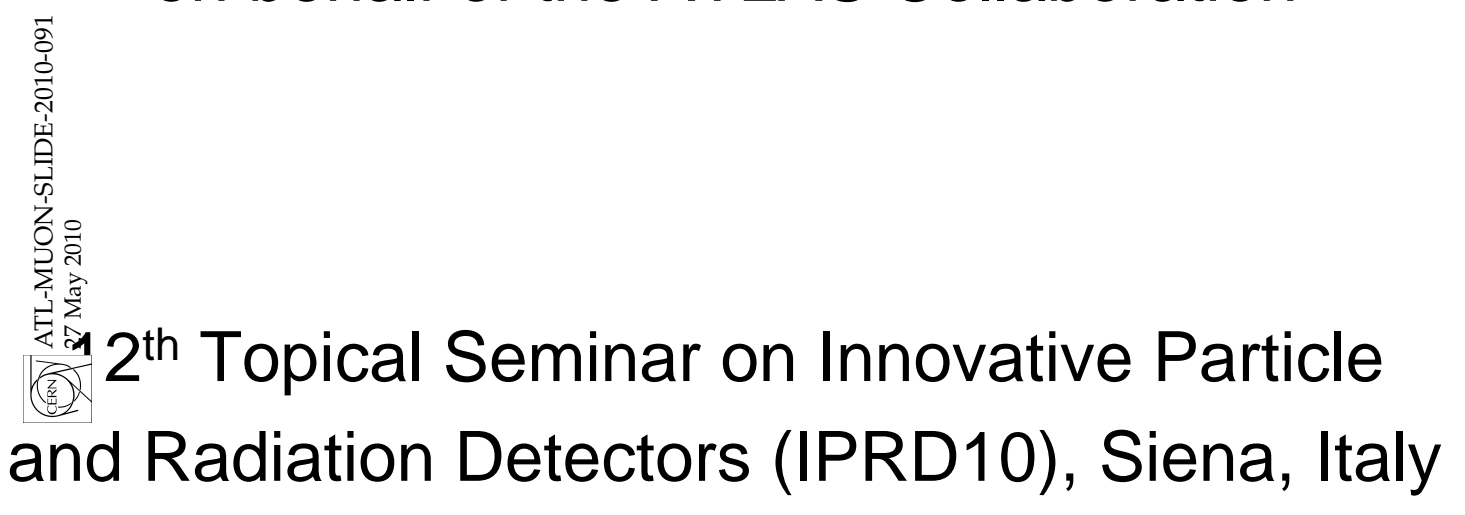

June 7-10, 2010 


\section{Outline}

- ATLAS Muon Spectrometer

- Muon calibration constants for muon reconstruction

- Determine muon drift tube timing offsets (TO) using beam splash events

- Determine time-to-space (RT) function using gas monitor chambers

- Validation of the calibration constants

- Conclusion 


\section{ATLAS Detector}

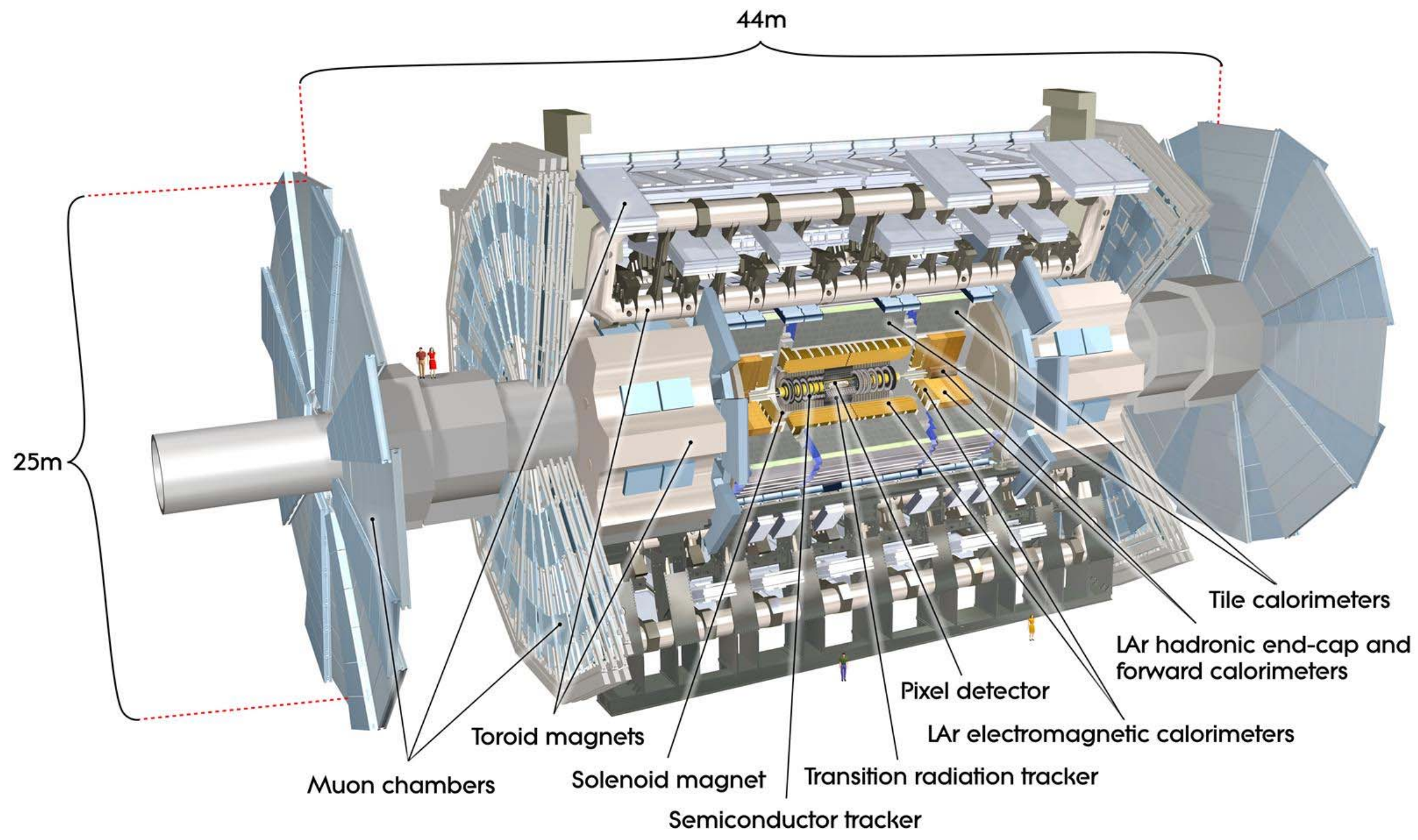




\section{ATLAS Muon Spectrometer}

1) Subsystem

$>$ MDT chambers are used for precisely measurement, with $<100 \mu \mathrm{m}$ precision; Barrel: BI, BM, BO; Endcap: El, EM, EO

$>$ CSC chambers exist in high- $\eta(|\eta|>2.0)$ region of the innermost station to cope with high rate measurement

$>$ Trigger chambers: TGCs and RPCs (second coordinate measurement)

$>$ Coverage $|\eta|<2.7$

2) The momentum resolution is typically $\sim 3 \%$ over most of the $\mathrm{Pt}$ ranges; at $\mathrm{Pt}=1 \mathrm{TeV}$ it is expected to be $10 \%$. (error dominated by calibration and chamber alignment)

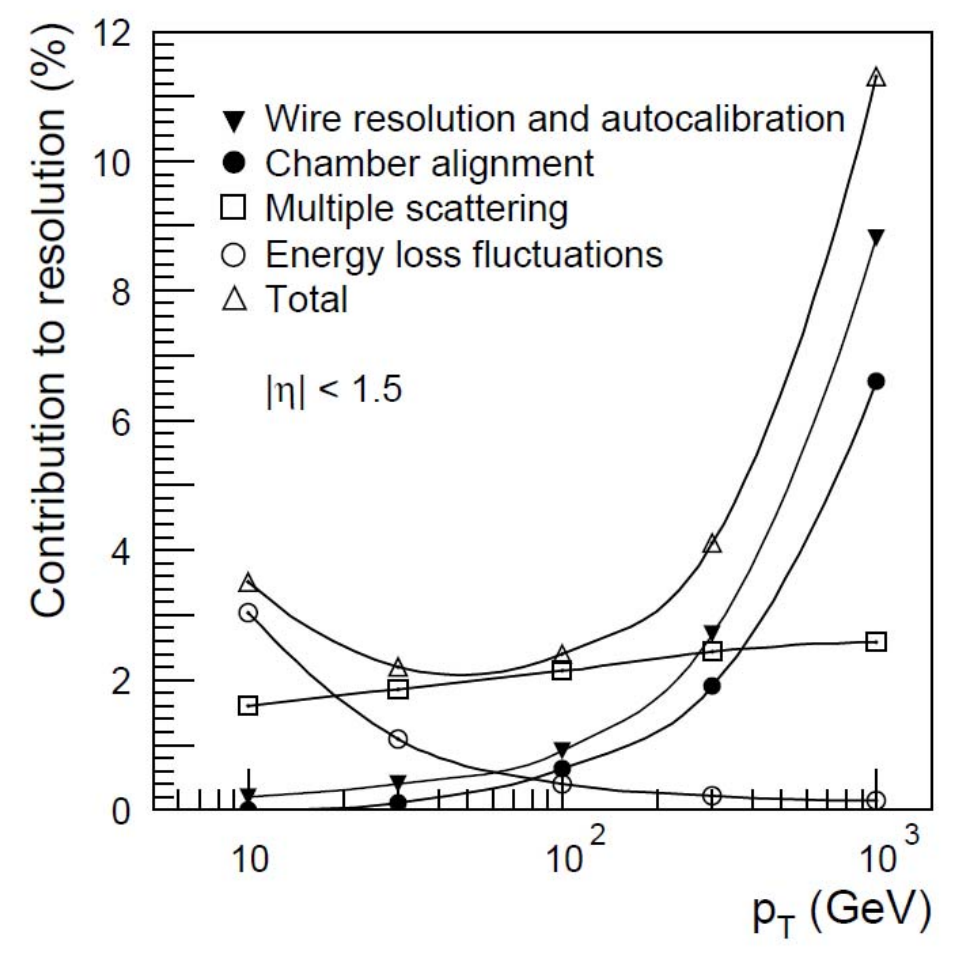




\section{Single Proton Beam in ATLAS (Nov. 2009)}

- Beam "splash" events with closed collimators $\rightarrow$ Beam 1 from ATLAS A side $\rightarrow$ Beam 2 from ATLAS C side

- All detector components were getting hits $\rightarrow$ Occupancy -100\%

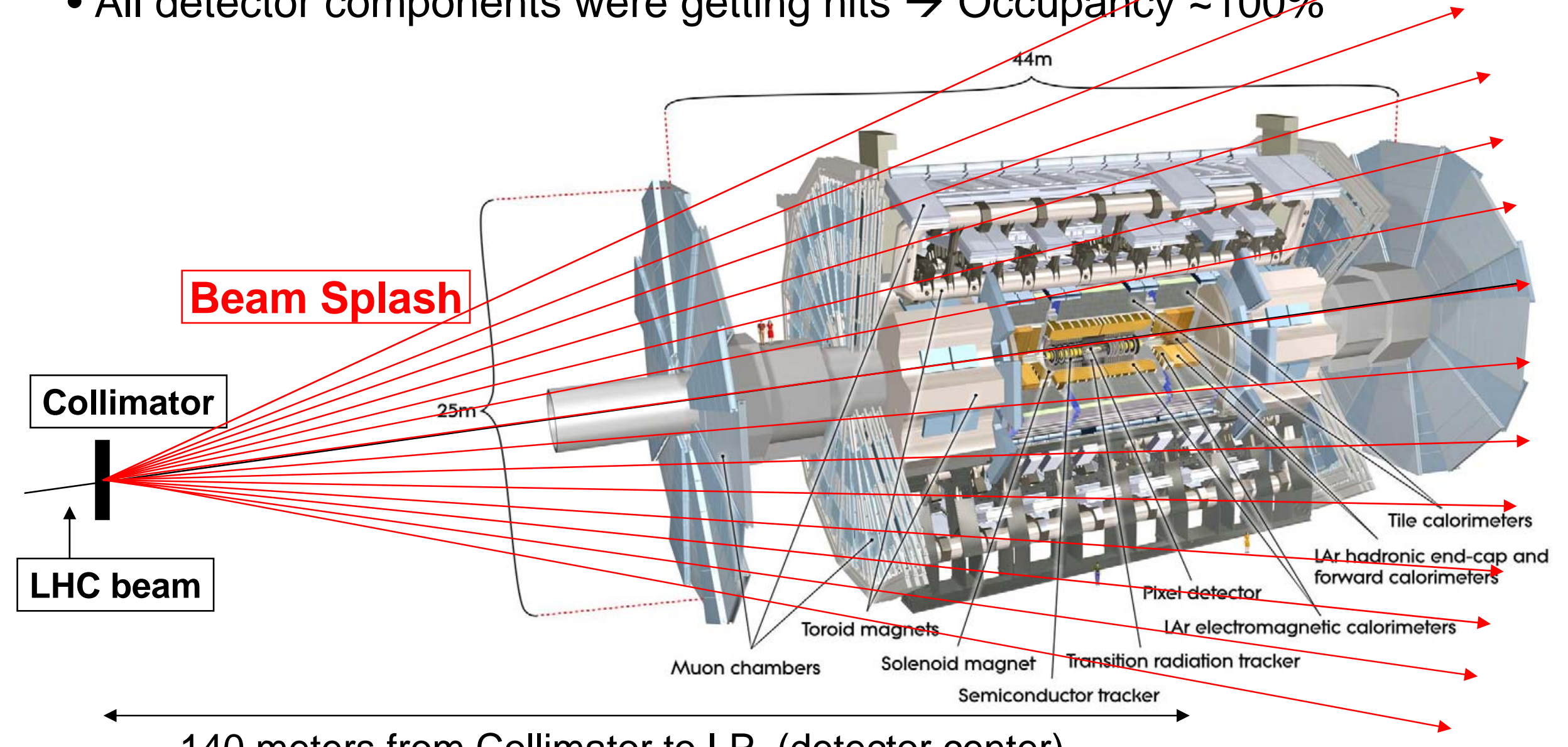
140 meters from Collimator to I.P. (detector center) 


\section{Beam Splash Events}

Beam intensity: $\sim 2 \times 10^{9}$ protons/bunch

MDT chamber occupancy 95\%

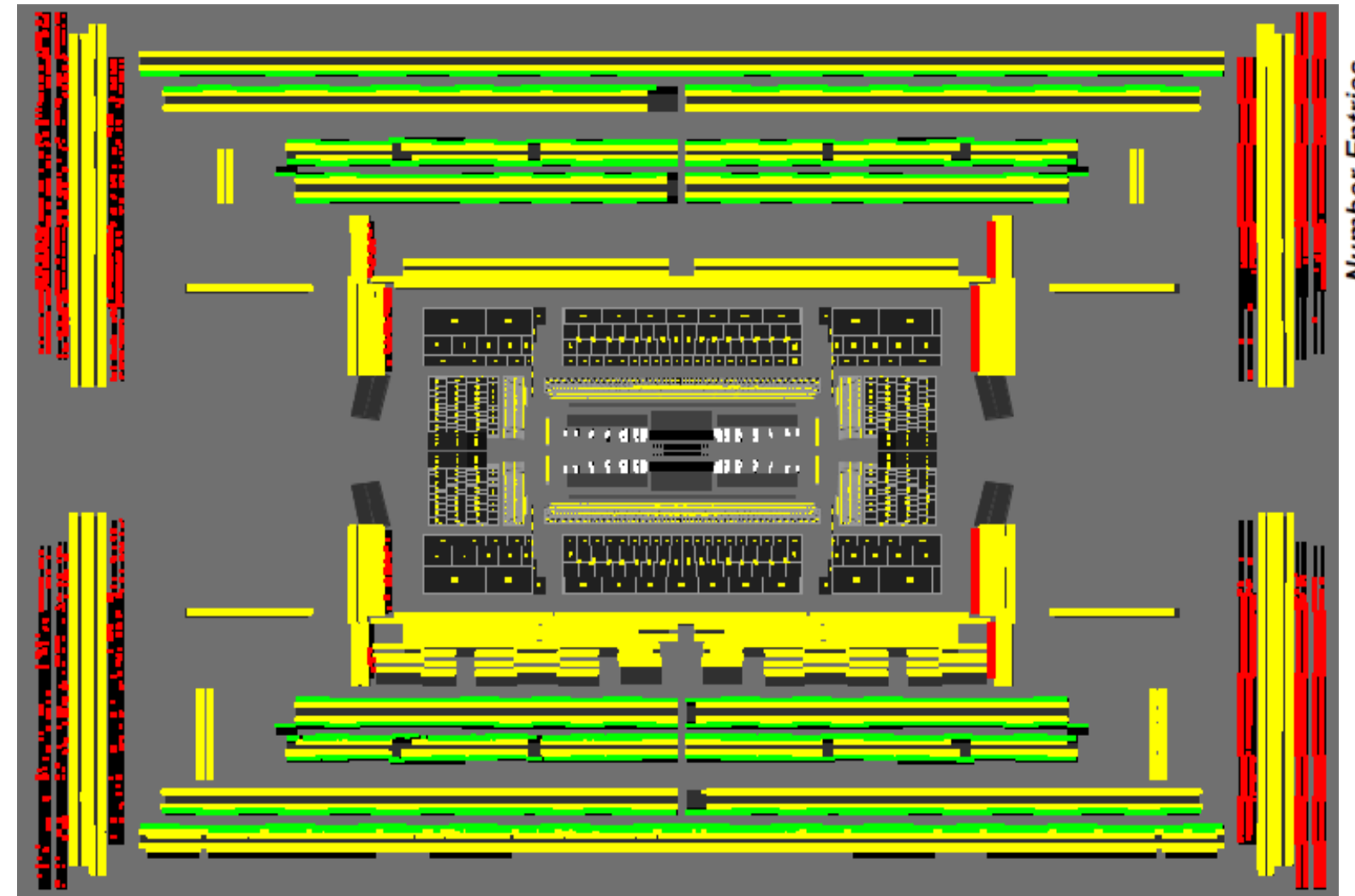

Recorded hits in projections of ATLAS Detector

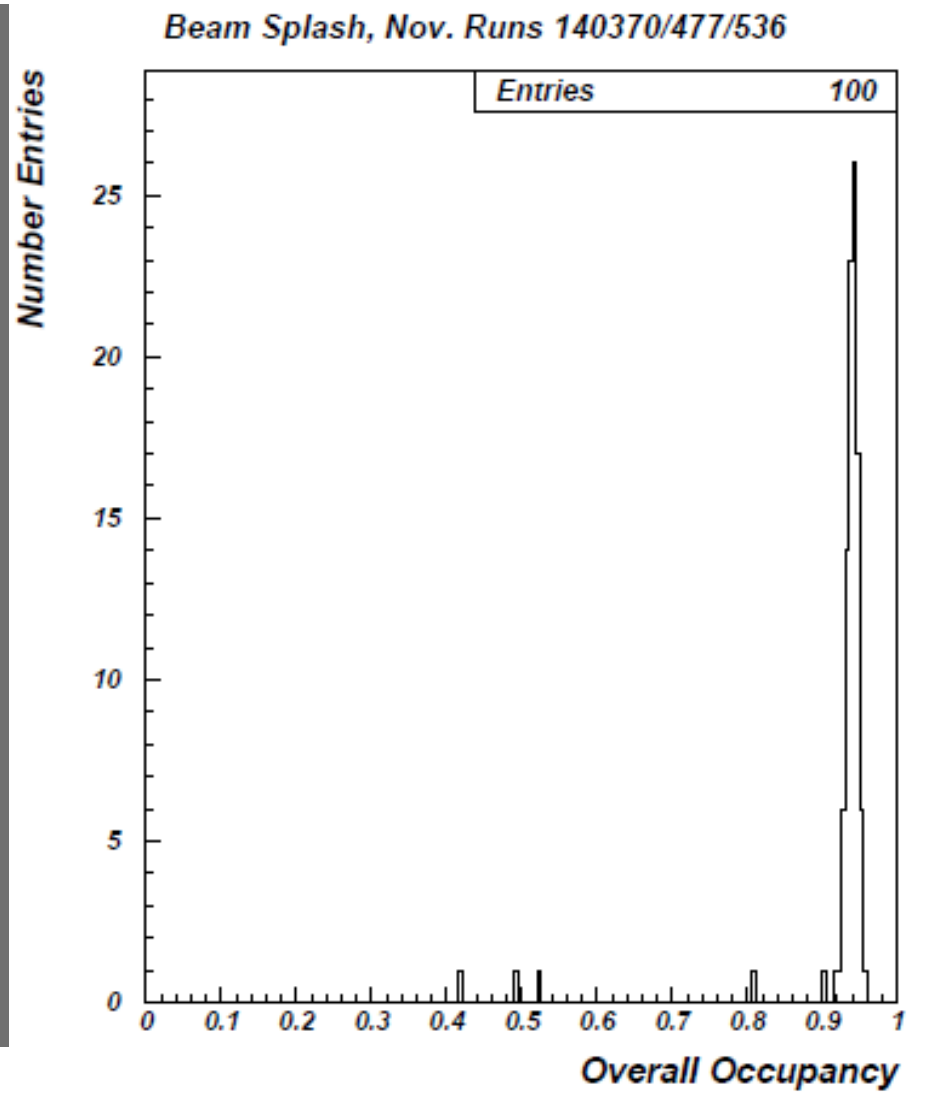




\section{Find Time Offset From Beam Splashes}

1) Use combined beam splash runs:

- 35 events for beam1 $(A \rightarrow C)$

- 59 events for beam2 $(C \rightarrow A)$

2) Analyze time spectra from Beam1 and Beam 2 separately

3) Use moving average to smooth spectrum \& find peak

4) Raw T0 is taken as TDC time for the bin which has $5 \%$ magnitude of the peak
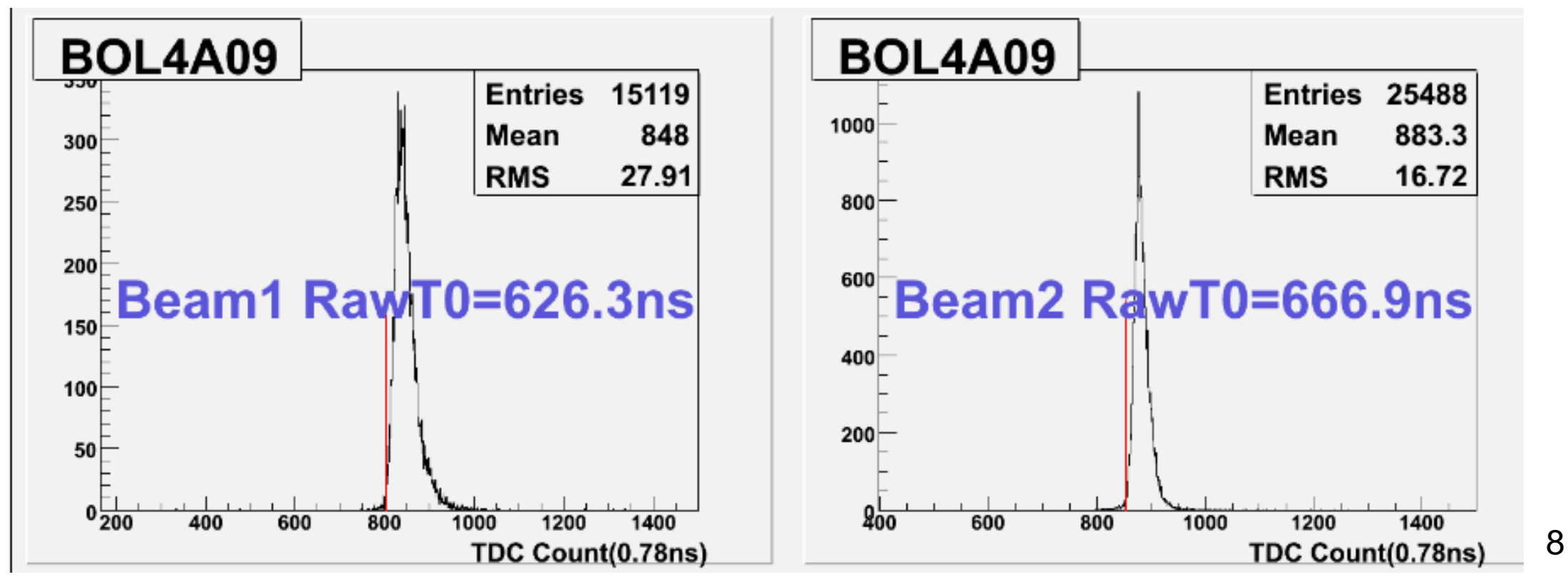


\section{Beam splash MDT TDC Spectra}
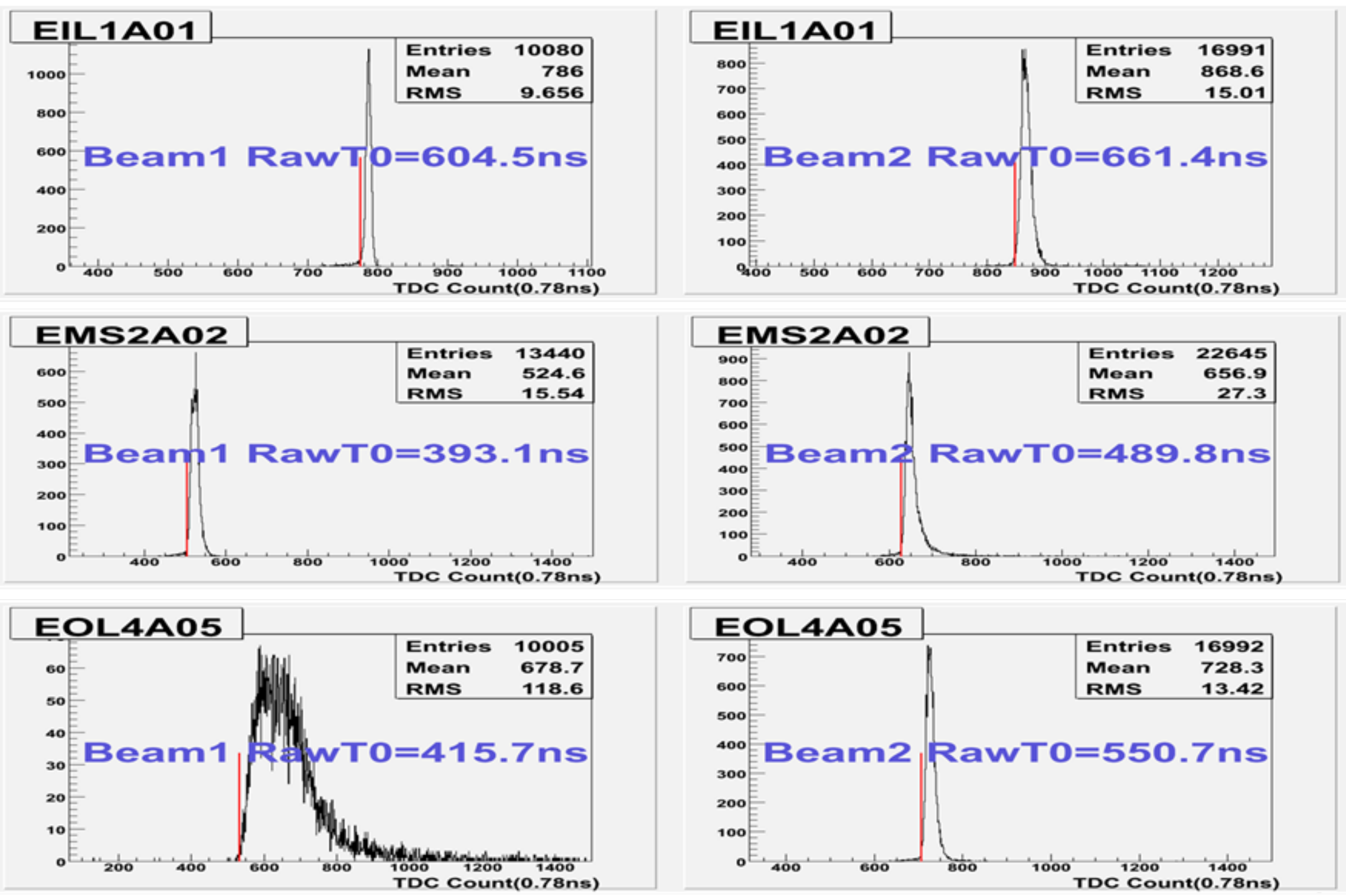


\section{Geometric Correction for Collision (Calculate T0 from Beam Splash Raw T0)}

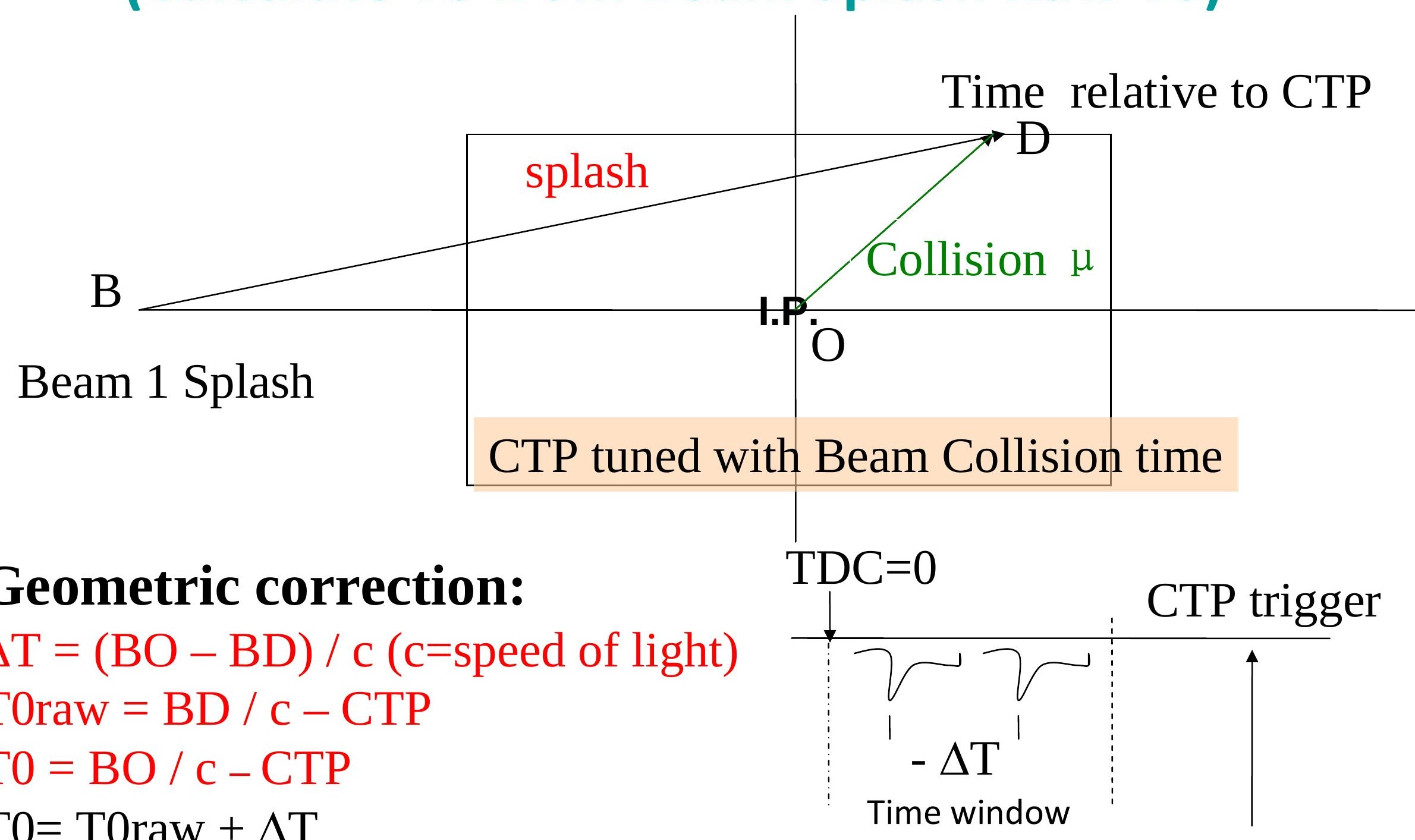




\section{Compare T0's from Beam1 \& Beam 2}

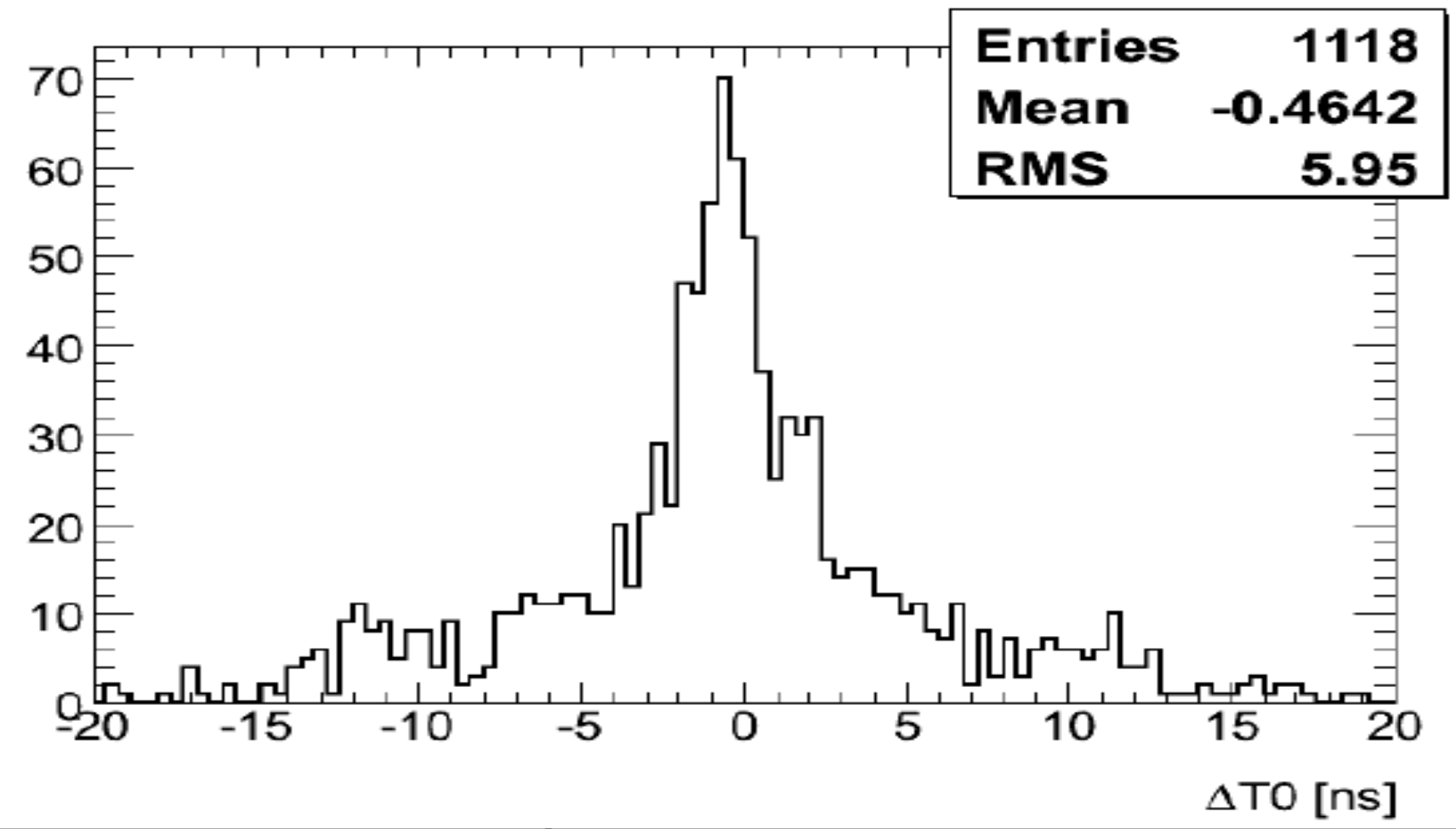

\begin{tabular}{|c|c|c|}
\hline T0 Difference (ns) & \# of Chambers & Percentage \\
\hline$<5$ & 698 & $62.77 \%$ \\
\hline$<10$ & 888 & $79.86 \%$ \\
\hline$<20$ & 1029 & $92.54 \%$ \\
\hline$>20$ & 83 & $7.46 \%$ \\
\hline
\end{tabular}




\section{Comparison T0's obtained in Nov. 2009 and in Feb. 2010 Using Beam Splash Events}
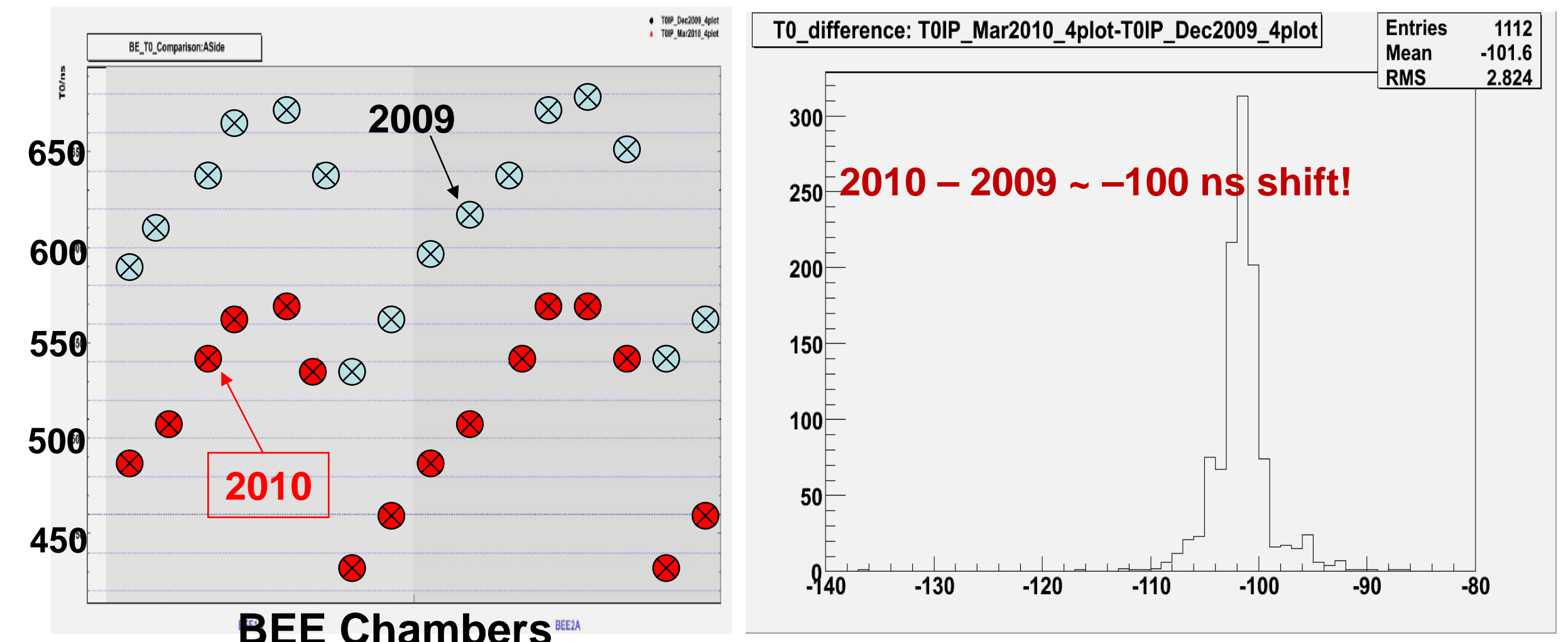

2010 Beam Splash TOs are systematically 100ns smaller than 2009 Beam splash TOs $\rightarrow$ due to LHC trigger 4 ticks shift (100ns) 


\section{Determine RT Using Gas Monitor Chamber}

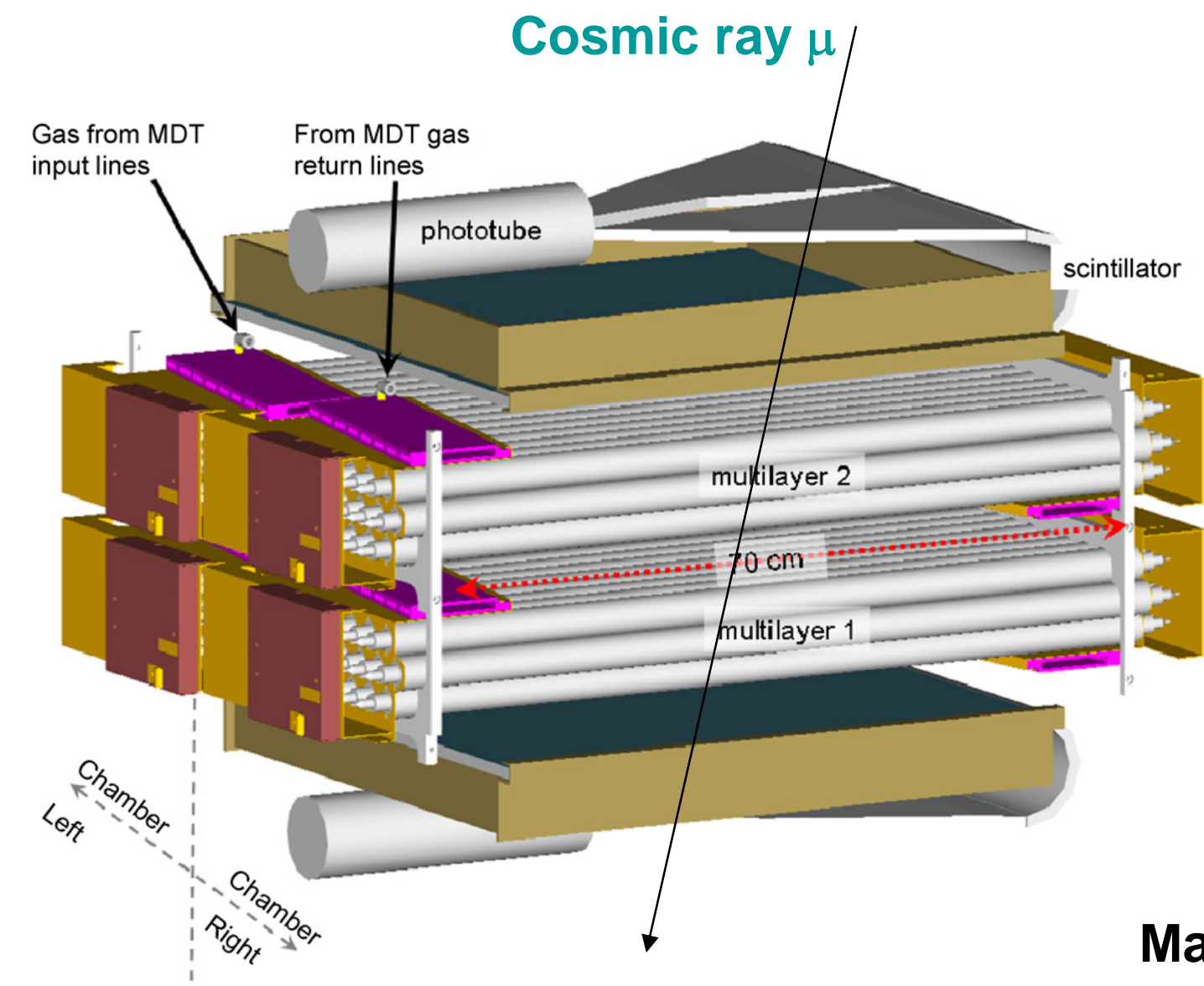

Monitor Max. Drift time

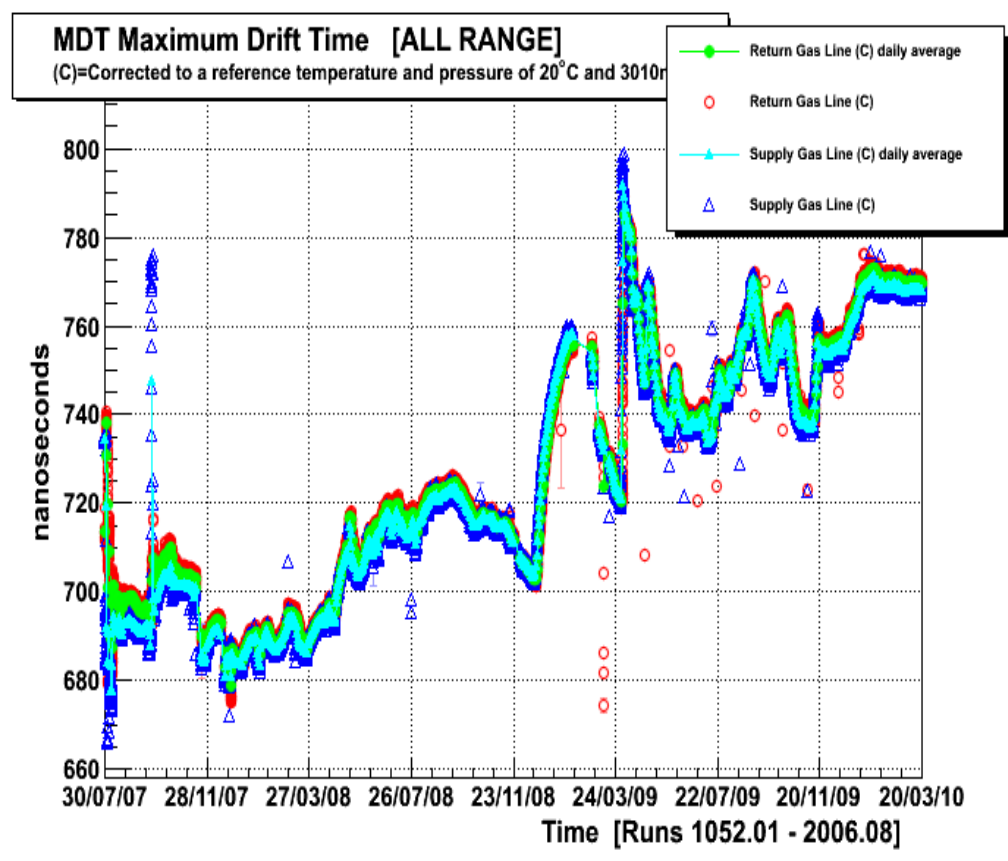

$\mathrm{P}=3$ bar with standard MDT gas mixture: $93 \% \mathrm{Ar}, 7 \% \mathrm{CO}_{2}$ which is sensitive to RT. It is determined by fit the TDC spectrum. Update RT every day. 


\section{$\mathrm{T}_{\max }$ Vs. Pressure, Temperature}
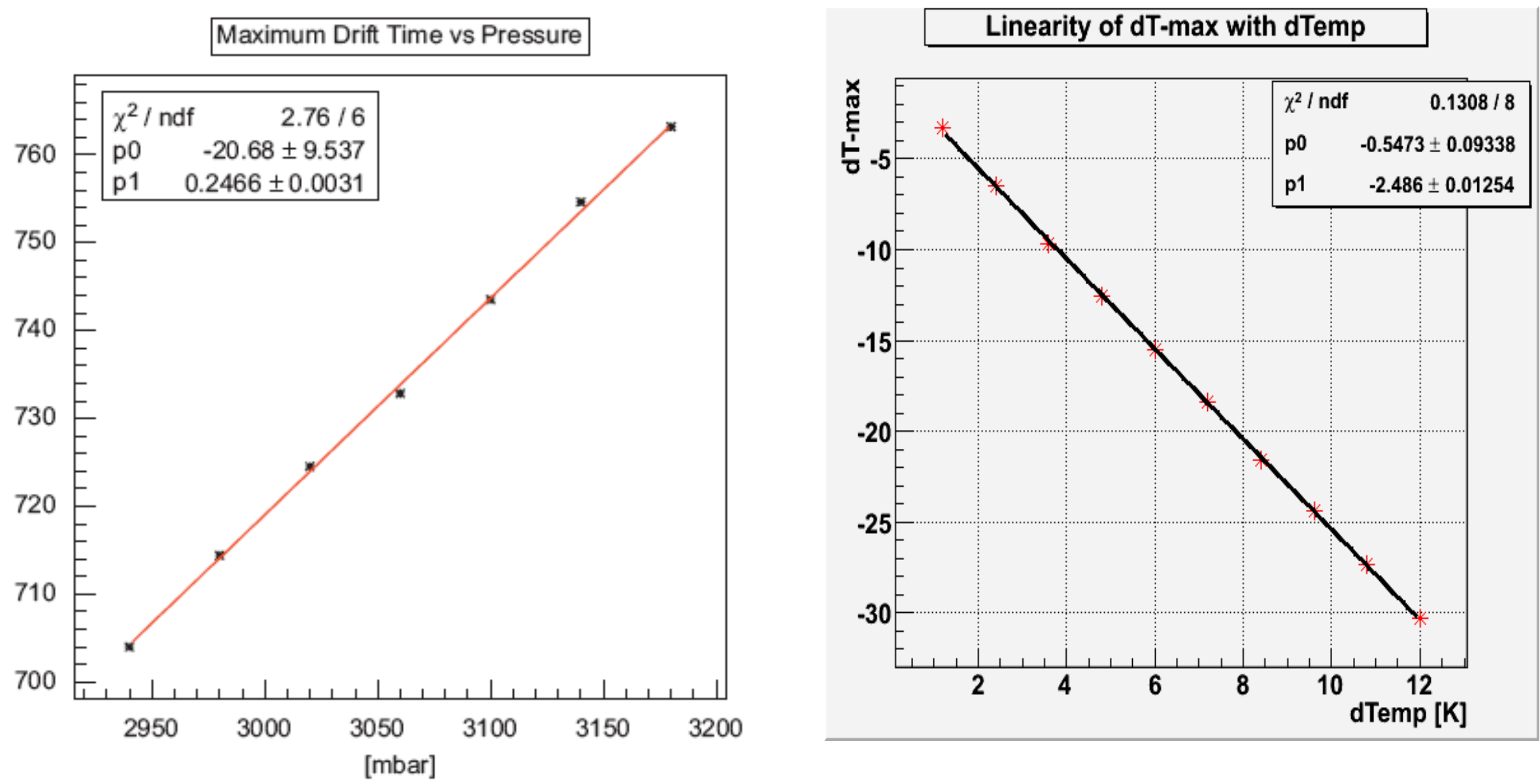

RT for each MDT chamber is corrected by Pressure and Temperature 


\section{MS Segment Reconstruction}

(with calibration constants)

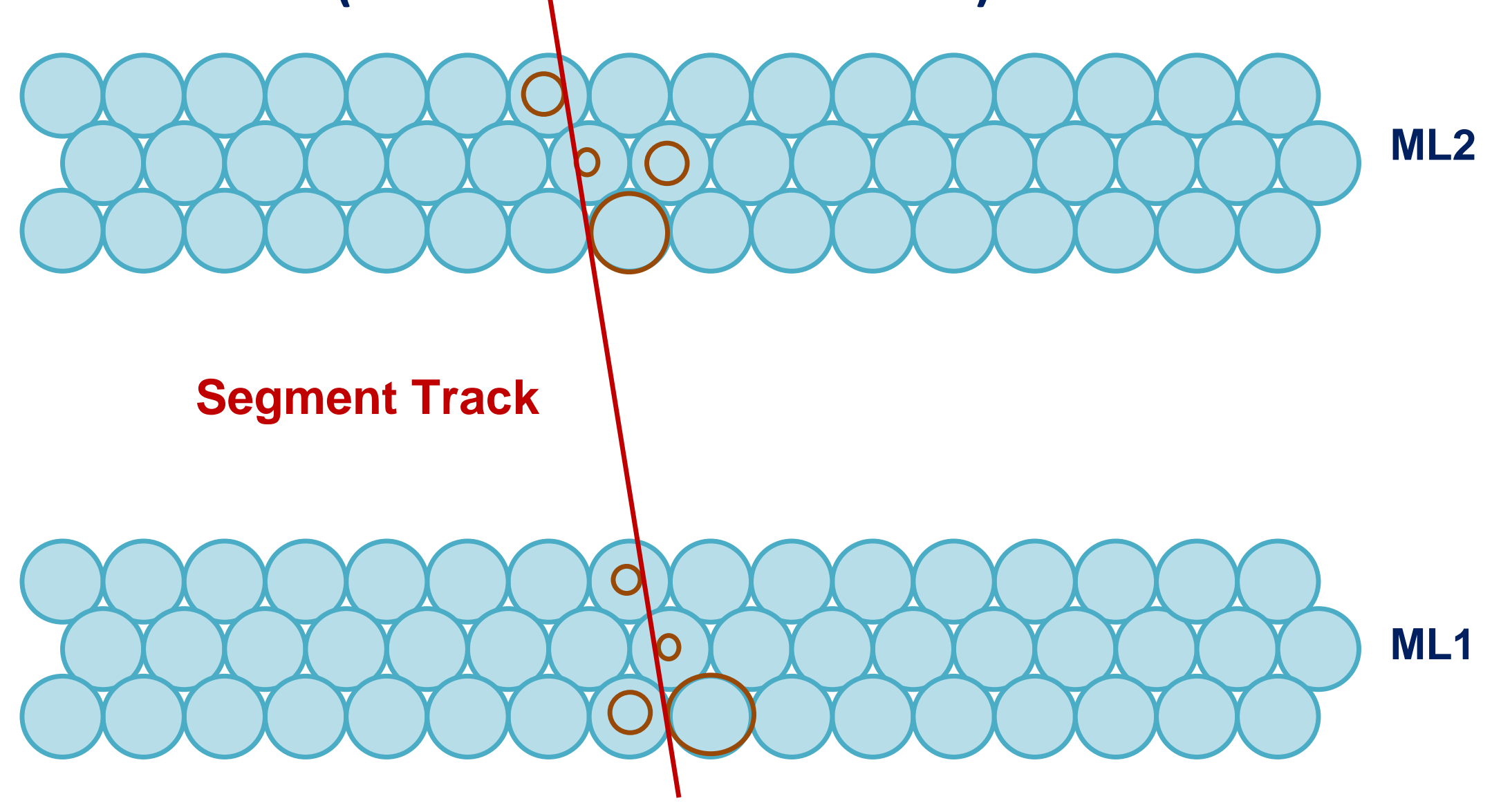

Using the time offset (TOs) and RT to determine the drift distance (circles) of each hit for track fitting 


\section{Validation of Muon Calibration Constants}

- Using beam collision muons to validate the T0s obtained from beam splash events.
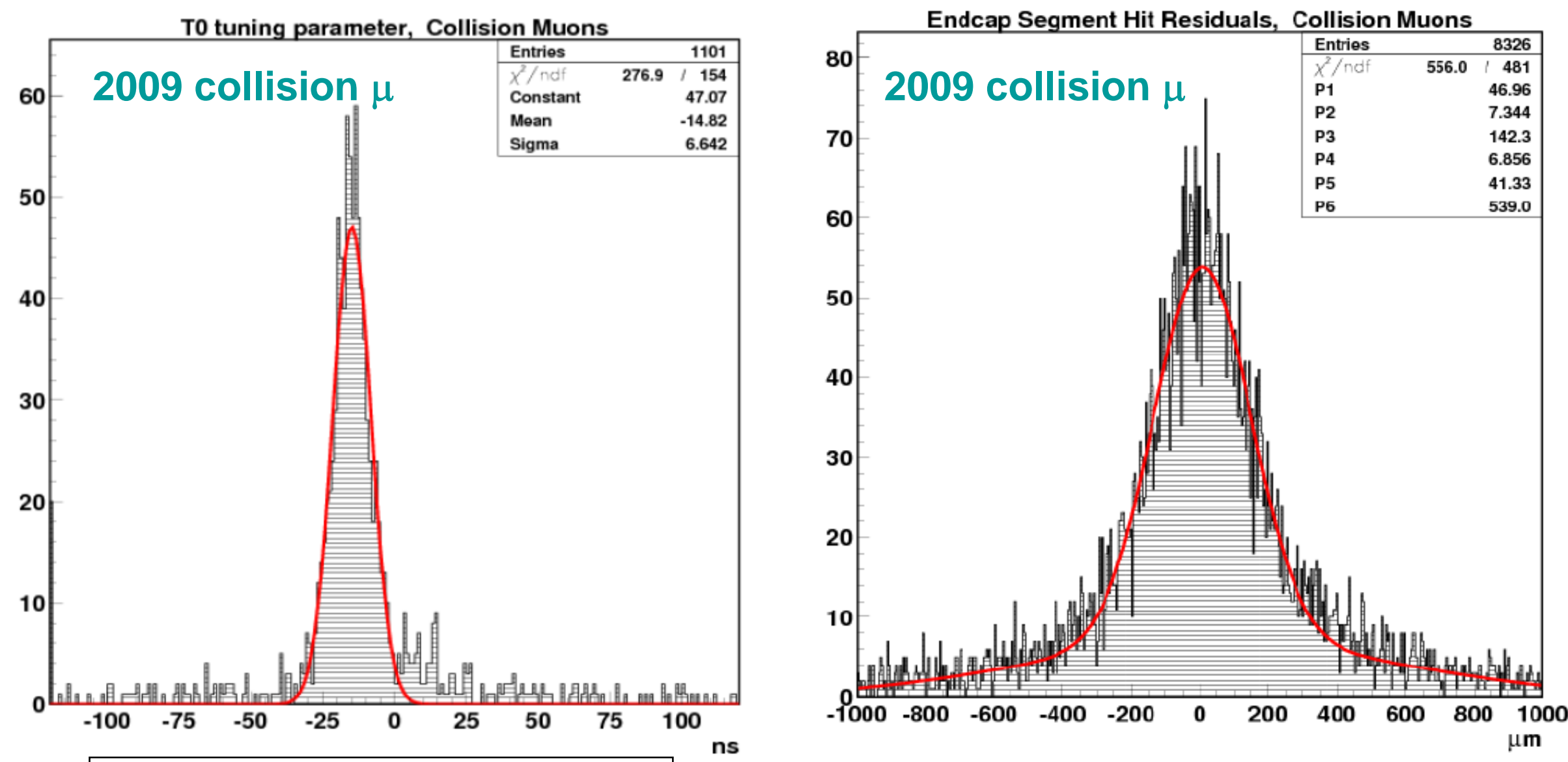

$\sim 14$ ns global offset of T0 from beam splash events 


\section{Validation of Muon Calibration Constants}

Using beam collision muons to validate the T0s obtained from the beam splash events and RT function using gas monitor chamber.

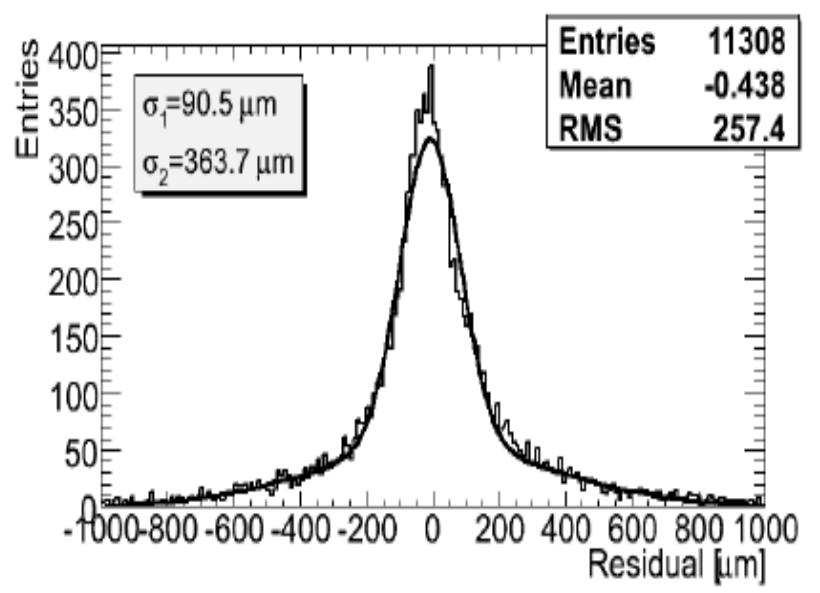

MDT Segment resolution $\sim 90 \mu \mathrm{m}$

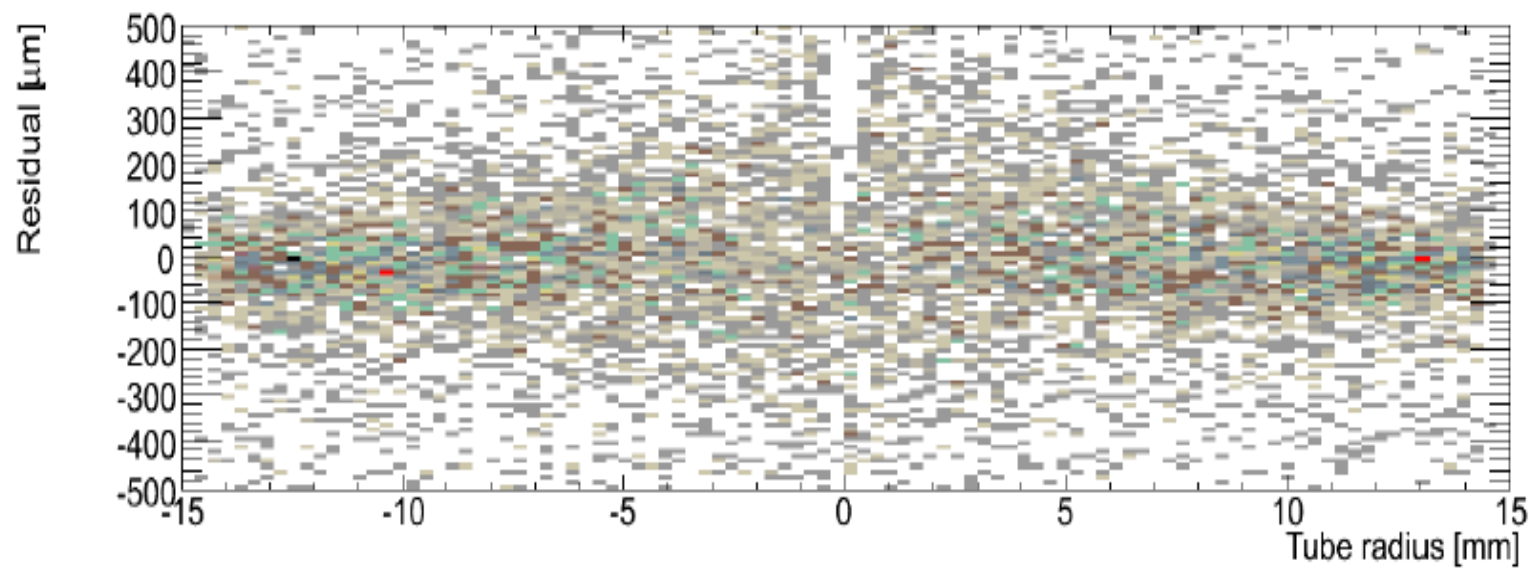

Segment residual vs drift radius

Validation with 2010 collision data, both residual distribution and residual vs. drift distance plots show that the calibration constants are accurate 


\section{Segment Track Fit Residuals}

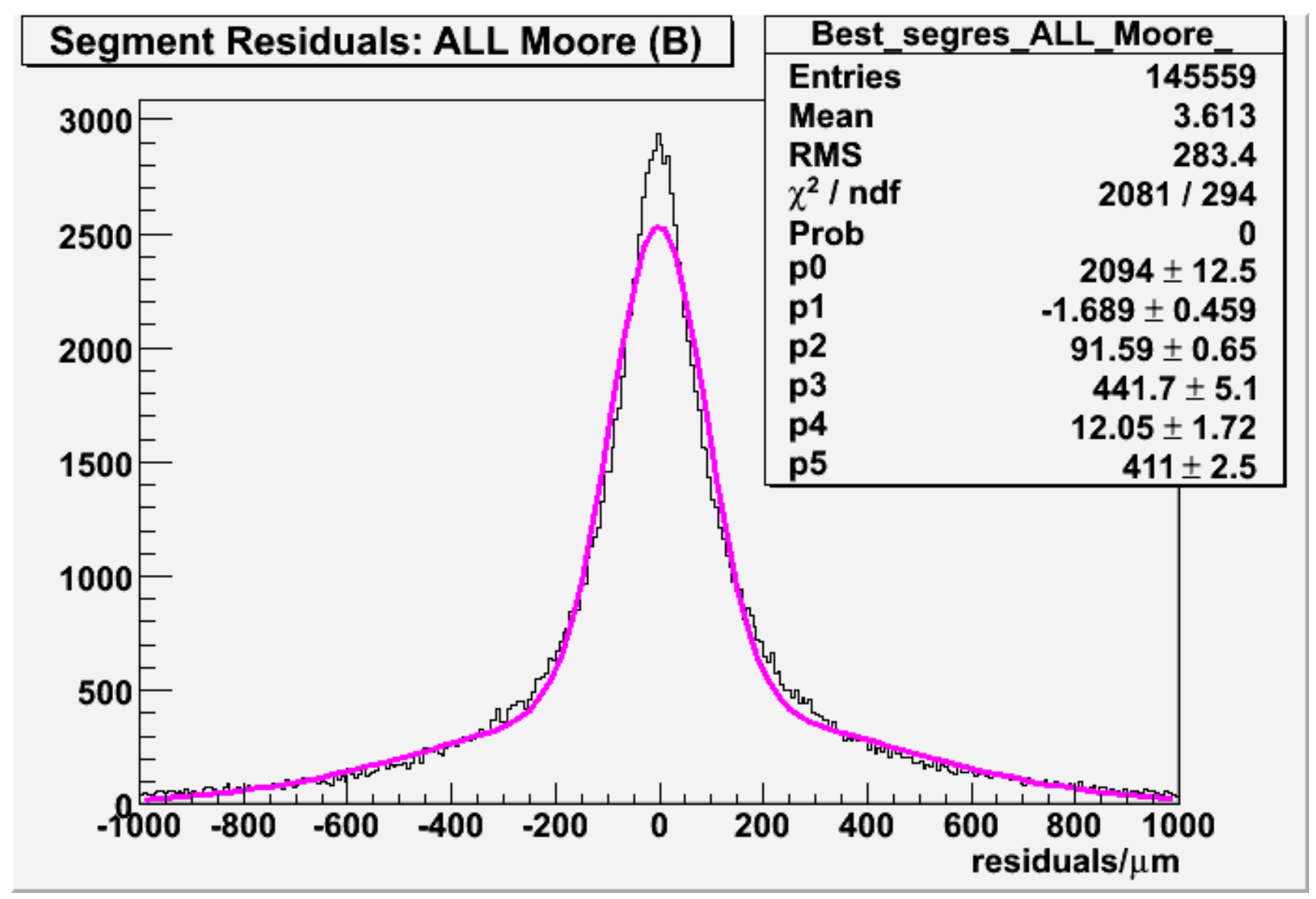

MDT segment track fit resolution $\sim 90 \mu \mathrm{m}$ 


\section{Validation using $\mathrm{J} / \psi \rightarrow \mu^{+} \mu^{-}$Mass Spectra from $7 \mathrm{TeV}$ Data}

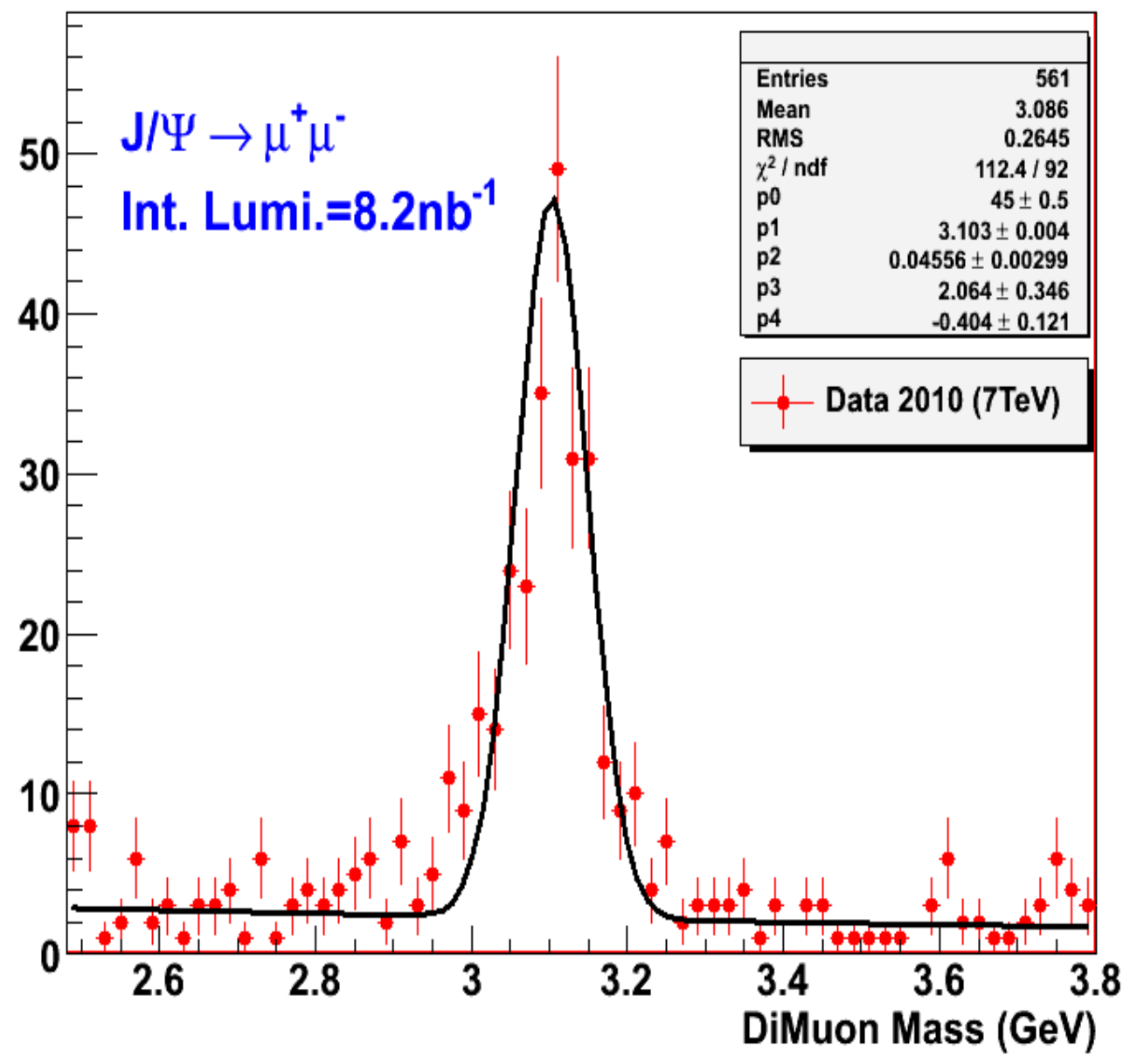

$\mathrm{J} / \psi \rightarrow \mu^{+} \mu^{-}$mass spectra can determine muon momentum or energy scale at low Pt region.

Event are selected with at least one muon reconstructed by both inner tracker and MS.

mass $=3.103 \pm 0.004 \mathrm{GeV}$ resolution $=46 \pm 3 \mathrm{MeV}$

It indicates that the initial muon calibration constants are valid. 


\section{Conclusions}

- ATLAS Muon MDT Calibration constants timeoffset (TO's) have been determined by using beam splash events with an accuracy $\sim 3 \mathrm{~ns}$.

- The universal MDT RT function has been determined by the gas monitor chamber. This function has been used in muon reconstruction with temperature and pressure corrections for each MDT chamber.

- Using the muon tracks from the initial LHC beam collisions, the MDT calibration constants have been validated $\rightarrow$ the tracking residual $\sigma \sim 90 \mu \mathrm{m}$. 